\title{
RPF: An Extensible, Cross-Platform, Binary File Format for Radiation Physics Data
}

A Compendium of Technical Work papers

Cheryl L. Ham

September 10, 2002

U.S. Department of Energy

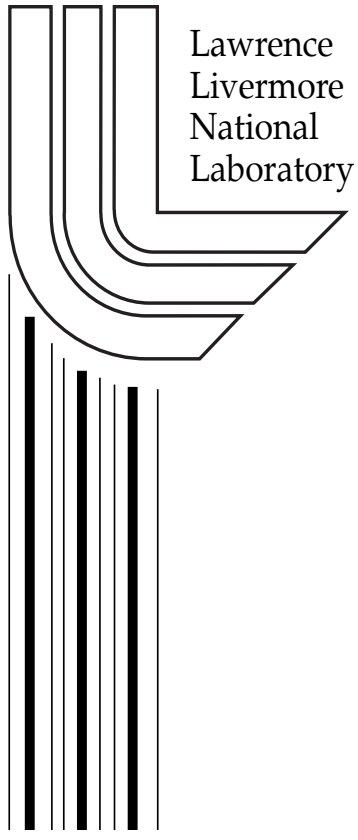




\section{DISCLAIMER}

This document was prepared as an account of work sponsored by an agency of the United States Government. Neither the United States Government nor the University of California nor any of their employees, makes any warranty, express or implied, or assumes any legal liability or responsibility for the accuracy, completeness, or usefulness of any information, apparatus, product, or process disclosed, or represents that its use would not infringe privately owned rights. Reference herein to any specific commercial product, process, or service by trade name, trademark, manufacturer, or otherwise, does not necessarily constitute or imply its endorsement, recommendation, or favoring by the United States Government or the University of California. The views and opinions of authors expressed herein do not necessarily state or reflect those of the United States Government or the University of California, and shall not be used for advertising or product endorsement purposes.

This work was performed under the auspices of the U. S. Department of Energy by the University of California, Lawrence Livermore National Laboratory under Contract No. W-7405-Eng-48.

This report has been reproduced

directly from the best available copy.

Available to DOE and DOE contractors from the

Office of Scientific and Technical Information

P.O. Box 62, Oak Ridge, TN 37831

Prices available from (423) 576-8401

http:/ /apollo.osti.gov/bridge/

Available to the public from the

National Technical Information Service

U.S. Department of Commerce

5285 Port Royal Rd.,

Springfield, VA 22161

http://www.ntis.gov/

OR

Lawrence Livermore National Laboratory

Technical Information Department's Digital Library

http://www.llnl.gov/tid/Library.html 
University of California

Lawrence Livermore National Laboratory

Technical Information Department

Livermore, CA 94551

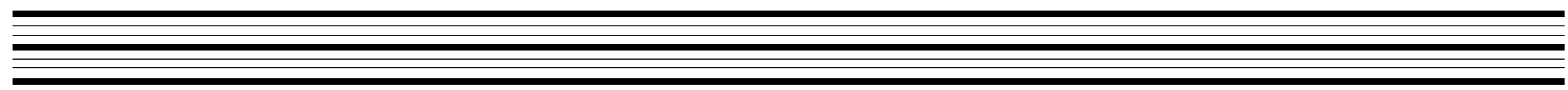




\title{
RPF: An Extensible, Cross-Platform, Binary File Format for Radiation Physics Data
}

\author{
A Compendium of Technical Work Papers
}

Cheryl L. Ham

Lawrence Livermore National Laboratory

\section{Contents \\ Introduction \\ RPF Spectral Data Format \\ RPF Software Suite Overview \\ RPFTools Reference Manual \\ Data Dictionary: Header Keywords for the Radiation Physics Format 24 \\ RPF Future Directions 34 \\ Appendix A: An Introduction to the Common Data Format (CDF) \\ Appendix B: About HDF and Supported Platforms \\ 1 \\ 3

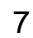

\section{Introduction}

Lawrence Livermore National Laboratory's Radiation Technology Group (RTG) uses a number of computer codes for simulation and analysis of radiation data. The number of incompatible data formats that these data presented themselves in have continued to multiply. In the 1980's a Common Data Format (CDF, see Appendix A) was devised for internal use by the RTG. This format represented a single gamma-ray spectrum as ASCII energy/count pairs preceded by an ASCII header. The ASCII representation of the data assured that it was compatible on any computing platform and this format is still in use.

In the mid 1990's it became apparent that instrument systems of greater complexity would demand a file format of larger capacity to support systems then on the drawing board, including networks of sensors collecting time series of gamma-ray spectra. These systems were in the planning stage and defined data structures were not available. It became apparent that a new storage format for nuclear measurements data would be needed and it would have to be flexible and extensible to accommodate the requirements of systems of the future. As part of an LDRD, we began to investigate what others were doing, especially in the high-energy physics community, to deal with the large volumes of data being generated.

Of particular interest was the very general Hierarchical Data Format (HDF), developed and maintained by the National Center for Supercomputing Applications (NCSA), that we ultimately used to develop the Radiation Physics Format (RPF). The HDF subroutine library provides users with the ability to customize a data file format based on standard calls to the HDF subroutine library. The RPF was developed and deployed on Sun and Hewlett-Packard workstations running their proprietary versions of UNIX. 
An RPF spectral data file format consists of one or more data pages. A data page is composed of a header and its associated spectral data. It was implemented using the HDF vset paradigm which establishes a logical grouping of diverse, but related data items using only two basic types of storage elements: vgroups and vdatas. Vgroups contain only references to vdatas or other vgroups. Vdata contain only data.

Reading, writing, and printing RPF data files is accomplished by calls to the RPFTools library, written by the author, that in turn relies on calls to the HDF subroutine library. The RPFTools library, developed in ANSI C, currently contains 18 functions for reading, writing, and printing RPF data files. Its dependencies are the HDF package, the standard C libraries, and clhtools, which is a collection of miscellaneous ANSI C utility functions, also written by the author.

In addition to the RPFTools, two stand-alone programs were written that illustrate the use of the library: cdf2rpf translates a CDF data file into an RPF file and showrpf allows a user to open and browse the contents of an RPF file.

It is important to summarize the fact that the RPFTools do not, of themselves, constitute a finished data format. They are tools for the creation of application-specific radiation physics data formats that can be structured to satisfy a user's requirements. This means that once such a structure is defined, some code must be written, using the RPFTools, to read, write, and print out the user's data. The RPFTools make the creation of that code a relatively simple task and the user can be assured that his or her data can be readily transferred intact and be ready to use on other computing platforms.

Upon reaching the stage of development described in this document, the RPFTools code suite was frozen at HDF version $4.1 \mathrm{r} 3$ pending the identification of a specific need. As a result, no final report was produced. This document is an unedited compendium of informal technical work papers produced by the author during various stages of the project. As a result some of the material is repetitive. Nevertheless, while informal, these papers give a view of the RPF at considerable technical depth. 


\section{RPF Spectral Data File Format}

History

Requirements
Neutron and gamma-ray spectrometry experiments can produce a large volume of data. At various times, these data need to be collected, annotated, collated, analyzed, transported, archived, and retrieved. In practice, these operations often occur on multiple platforms.

We needed a data file format that was flexible in the type and amount of data it could store, portable across different computer platforms, and was easily extensible to accommodate new data storage requirements. Finding an existing, well-developed framework that had freely distributed pre-compiled binaries for a wide variety of platroms; was open source for compilation on other platforms; had a large, diverse user base; and available user support was a bonus which helped to reduce the complexity and time required to implement a solution.

The Radiation Physics Format (RPF) spectral data file format is the result of designing a standard data file format for spectral data that meets these and other criteria. It is a selfdescribing binary file that is based upon the National Center for Supercomputing Applications (NCSA) Hierarchical Data format (HDF) paradigm.

This file format must accommodate neutron (count) data, gamma-ray spectral (energy, flux pairs) data, and statistical data. These data can be either collected from or calculated for any number of detectors as either a single count/spectrum or a time series of counts/spectra.

In addition, the data files must also accommodate varying types and amounts of information describing the origin, history, and contents of the data. It is desired that this header information be readable by the user.

These data files must also be flexible/extensible, and portable across various platforms in order to avoid the problem of converting vast quantities of legacy data when modifying/adding information to the files, or changing platforms.

The implementation of these data files is based upon ANSI C using as few systemdependent extensions as possible in order to promote cross-platform compatibility. Current platforms under consideration are UNIX (HP, Sun), PC (DOS and Windows), and Mac (Mac-OS).

A pre-existing, widely used, noncommercial basis for the data files gives the advantages of not having to develop low-level routines from scratch, low cost availability, a potentially large user base from which to draw utilities and expertise, and freedom from dependence on an inflexible commercial product and the longevity of any particular company.

The HDF file format package available from NCSA provides a good basis for a solution to our problem. (The HDF WWW home page is found at http://hdf.ncsa.uiuc.edu/. ) The NCSA anonymous ftp server is located at ftp.ncsa.uiuc.edu.) The following portion of 
this requirements section was excerpted from the beginning of the HDF FAQ and explains why the HDF framework was selected.

\section{What is HDF?}

What is in the HDF library?

Conceptual Solution
HDF stands for Hierarchical Data Format. It is a multi-object file format for the transfer of graphical and numerical data between machines.

HDF is a versatile file format. It supports six different data models. Each data model defines a specific type of data and provides a convenient interface for reading, writing, and organizing a unique set of data elements.

HDF is a self-describing format, allowing an application to interpret the structure and contents of a file without any outside information.

HDF is a flexible file format. With HDF, you can group sets of related objects together and then access them as a group or as individual objects. There are pre-defined sets for raster images and floating point multidimensional arrays. User can also create their own grouping structures using an HDF feature called vgroups.

HDF is an extensible file format. It can easily accommodate new data models, regardless of whether they are added by the HDF development team or by HDF users.

HDF is a portable file format. HDF files can be shared across platforms. An HDF file created on one computer, say a Cray supercomputer, can be read on another system, say IBM PC, without modification.

HDF is available in the public domain.

HDF currently supports six data structure types: 8-bit raster images, 24-bit raster images, color palettes, scientific data sets (multi-dimensional arrays), text entries and vdatas (binary tables).

The HDF library contains two parts: the base library and the multi-file library. HDF library functions can be called from $\mathrm{C}$ or FORTRAN user application programs.

The base library contains a general purpose interface and six application level interfaces, one for each data structure type. These application level interfaces are specifically designed to read, write and manipulate one type. The general purpose interface contains functions, such as file I/O, error handling, memory management and physical storage.

The multi-file part integrates netCDF model with HDF Scientific data sets, and supports simultaneous access to multiple file and multiple object. This part is referred to as netCDF/HDF library in the rest of this FAQ.

Our previous attempt at implementing a spectral file format, the CDF file, provides a good starting point. A CDF file is an ASCII file which has a variable length and content header consisting of keyword labels followed by value (e.g. nchan=4096) delimited by the keywords ::beginheader:: and ::endheader $::$ followed by the spectral data. The 
Specifications for the Actual Solution spectral data could consist of up to five columns of information. More than one spectrum could be sequentially stored in one CDF file.

The advantages of this solution is that the header is variable length, variable content, and easy to read, write, and modify by an application and by the user with any text editor. This also leads to the disadvantages of non-standard keywords and that the user can easily corrupt the file.

The major disadvantage to CDF is the amount of storage space that the data can consume.

The RPFTools software suite, and a couple of simple applications illustrating the suite, were written debugged and deployed on HP-UX, Solaris, and MacOS. A port to Windows was never completed. The suite was implemented using ANSI C standard code within the HDF version 4 framework. It is a binary variation on the CDF file solution in which there is a variable length, variable content header describing the HDF file content, and one or more sets of data with a variable length, variable content header describing the data.

The headers can be thought of as metadata since they are data about data and are implemented as HDF vgroups. The data file header consists of a variable number of vdatas that describe the data. The vdata's name can be thought of as its description or variable name. The vdata's class is its category. And the vdata's data are its values. For example, one header vdata entry might have a name of location, a class designation of experiment_identification, and a data value of measurement lab.

Data are represented by default as 16-bit integers or 64-bit floating point numbers depending upon their requirements unless specified otherwise by the user. Floating point numbers are automatically converted from the native format of the host machine to the standard HDF format of IEEE 32- or 64-bit floating point format as required. This is to promote cross-platform portability, a feature that is hindered by storing data using the native format option. Neutron data are stored as scientific data set (SDS) arrays of rank 3 to allow fields for time, neutron count, and detector. Gamma spectra are stored as SDS arrays of rank 4 to allow fields for time, energy, flux and detector. Calculated gamma spectra can have additional rank for relative error, absolute error, etc., if desired.

The use of the HDF model places a hurdle before the user who wants the advantages of an easily editable ASCII header. An HDF data file is binary. Tests performed on an annotated HDF data file show that the ASCII strings of the annotations are visible and comprehensible with listing utilities such as UNIX more and with text editors such as emacs. While RPF annotations are in order, they are spread throughout the RPF data file rather than being contiguous in any one place in the file. If you want to hand edit the string, you can. However, if you substitute another string with a different length, the whole HDF data file becomes unreadable. A similar situation has occurred many times in the past with CDF files. The HDF hurdle performs a useful service by encouraging the user to make header modifications with a more disciplined approach.

The disciplined approach is to create utilities for viewing, extracting, and replacing the header information. A viewing utility simply opens the RPF data file, extracts the header 
Keyword Integrity 


\section{RPF Software Suite Overview}

Introduction

\section{Anatomy of an RPF file}

The Radiation Physics Format (RPF) spectral data file format is an extensible, selfdescribing, binary data file format that can accommodate multiple sets of nuclear data within a single file and is portable across computing platforms. It is based upon the Hierarchical Data Format (HDF) software package developed and supported by the National Center for Supercomputing Applications (NCSA).

The RPFTools Software Suite comprises a variety of tools to initialize, read, write, view, and manipulate RPF data files. It contains source code, include files, linkable object modules of RPF-related and other miscellaneous functions, and stand-alone programs. It is written in ANSI standard C and links against the standard C I/O libraries and INCAS's HDF version 4.x libraries. Standalone programs exist to view RPF files and to create RPF files from existing CDF files. Tools for file conversion from other data formats can be made by writing programs using the appropriate functions in the RPFTools Software Suite.

An RPF spectral data file format consists of one or more data pages. A data page is composed of a header and its associated spectral data. It is implemented using the HDF vset paradigm which establishes a logical grouping of diverse, but related data items using only two basic types of storage elements: vgroup and vdata. Vgroups contain only references to vdatas or other vgroups. Vdatas contain only data.

The headers can be thought of as metadata since they are data about data. They comprise a variable number of keywords selected from a pre-established list of valid keywords and their values. The data page header is a vset that contains a variable number of vgroups and vdatas that describe the data. The vdatas name can be thought of as its description or variable name. The vdata's class is its category. And the vdatas data are its values. For example, one header vdata entry might have a name of location, a class designation of experiment_identification, and a data value of measurement lab.

Data are represented by default as 32-bit integers or 64-bit floating point numbers depending upon their requirements unless specified otherwise by the user. Floating point numbers will automatically be converted by the underlying HDF package from the native format of the host machine to the standard HDF format of IEEE 32- or 64-bit floating-point format as required. This is to promote cross-platform portability, a feature that is hindered by storing data using the native format option.

Neutron data are stored as Scientific Data Set (SDS) arrays of rank 3 to allow fields for time, neutron count, and detector. Gamma spectra converted from the locally developed Common Data Format (CDF is an early Radiation Technology group data file format) data files are stored as SDS arrays of rank 3 to allow fields for bin edge energy, bin center energy, and flux. Rank can be increased to allow fields for time and detector. Calculated gamma spectra can have additional rank for relative error, absolute error, etc., if desired.

RPF data can originate as instrumental data or computational simulations of experiments from single detectors, detector arrays, and networks of disparate detectors. Total 
Platforms

RPFTools library

count data can be stored as a time series or as multichannel scaling. Spectral data can currently be stored as pulse-height spectra. Time-stamped list mode storage for spectral data is planned for a later version. Data can be mixed within a single RPF file. Time series of spectra or gross count data from multiple detectors can be stored within a single RPF file. This would allow, for example, disparate measurements from a series of related experiments or from arrays of detectors from a network. RPF is an important new contribution to our core competency and has broad applicability.

The RPFTools Software Suite was initially developed on an HP 9000 model 735 running HP-UX v 9.01 using the HDF version 4.0r2 release that was precompiled for HPUX v 9.03. The current version, v2.2, was developed on the same HP platform using the HDF version 4.1b1 release that was precompiled for HPUX v 9.03.

The RPFTools Software Suite has also been ported to a Sun SPARCstation 10/512 running Solaris 2.5.1 using the HDF version 4.0r2 release that was precompiled for Solaris 2.4. The current version is v1.0b2.

It has been demonstrated that an RPF file created on the HP or Sun can be transferred to the Sun or HP, respectively, and viewed.

The RPFTools library, rpftools.o, is generated from the source code module, rpfools.c, written in ANSI C. It currently contains 18 functions for reading, writing, and printing RPF data files. Its dependencies are the HDF package, the standard C libraries, and clhtools, which is a collection of miscellaneous ANSI C utility functions.

Function name init_rpf_header

reset_rpf_header

free_rpf_header

init_rpf_header_status

add_rpf_comment

parse_cdf_header

print_current_rpf_header

print_full_rpf_header

read_open_rpf_file

write_open_rpf_file

close_rpf_file

read_rpf_header

\section{Function description}

Initializes the space to hold the RPF header information and returns a pointer to the new RPF header data structure.

Resets the RPF header values to initialized values.

Frees the space used by the RPF header.

Initializes the space to hold the RPF header status and returns a pointer to the RPF header status data structure

Adds a string to the RPF header comment field.

Parses the header of a local-CDF file and fills in the corresponding variables in a RPF header.

Prints the portion of the RPF header that is currently valid to stdout.

Prints the full RPF header to stdout.

Opens an RPF file for reading.

Opens an RPF file for writing.

Closes an RPF file.

Reads an RPF header from an RPF data file. 


\begin{tabular}{|c|c|}
\hline write_rpf_header & Writes an RPF header into an RPF data file. \\
\hline read_rpf_data & $\begin{array}{l}\text { Reads the RPF data from an RPF data file and returns } \\
\text { pointer to the array of RPF data values. }\end{array}$ \\
\hline write_rpf_data & $\begin{array}{l}\text { Writes the RPF data into an RPF data file and returns the } \\
\text { reference number of the RPF data scientific data set } \\
\text { (SDS). }\end{array}$ \\
\hline print_rpf_data & Prints the RPF data to stdout. \\
\hline read_rpf_page & Reads an RPF page from an RPF file. \\
\hline write_rpf_page & Writes an RPF page to an RPF file. \\
\hline
\end{tabular}

Stand-alone applications

There are currently two standalone RPF applications in the RPFTools Software Suite. However, showrpf requires some additional debugging on the Sun.

\section{Program name \\ Program description}

cdf2rpf

Translates a CDF data file into an RPF file.

showrpf

Generates user-controlled display an RPF file to stdout. 


\section{RPFTools Reference Manual}

Introduction

Information Pages

\section{RPF Software Tools}

The Radiation Physics Format (RPF) spectral data file format is an extensible, selfdescribing, binary data file format which is portable across computing platforms. It is based upon the Hierarchical Data Format (HDF) software developed by the National Center for Supercomputing Applications (NCSA).

This document describes the Radiation Physics Format (RPF) spectral data file format and the software tools used to manipulate the RPF data files.

An RPF data file consists of one or more pages of information. A page consists of a header describing the spectral data plus the associated spectral data.

Each page of information is stored as an HDF vgroup in the RPF file. The vgroup class is rpf_page. The page name is generated by concatenating the string page_, the page index and the string "_" before it is stored as the vgroup name. There is a header vgroup and a data vgroup associated with each page vgroup. The vgroup classes of these are rpf_page_header and rpf_page_data, respectively.

The header vgroup consists of a variable number of keyword vdatas that encapsulate the header information. The name of the keyword vdata is the variable name in the corresponding RPF header data structure. The keyword vdata class is the name of the keyword category designation for that keyword inside the RPF header_type data structure. The keyword data fields are defined with the appropriate data type and named the same as the keyword vdata. Only those keywords which contain valid data as indicated by their corresponding rpfheader_status fields being set to ON will be written to the RPF file when the header is stored.

The data vgroup consists of a multidimensional data block which is stored as a scientific data set (SDS). The dataset name is generated by concatenating the string data_, the data index and the string ".." The data label attribute is set to gamma_data. The first three dimensions are labeled bins, units, and boxes, respectively to designate, single spectra, temporal series of spectra, and spectra gathered from array of detectors. Single spectra are normally concerned with energy bins. Temporal spectra are concerned with spectra taken within slices of time. A box of spectra would be those collected from an array of detectors. Alternatively, one may think of the second dimension as adding spectra and the third dimension as adding detectors.

The RPFTools software suite comprises of a variety of tools to initialize, read, write, view, and manipulate RPF data files. It contains source code, include files, linkable object modules of RPF related and other miscellaneous functions, and standalone programs. It is written in ANSI standard C and links against the standard C I/O libraries and NCSA's HDF version 4.3 libraries.

The include file, rpftools.h, defines a data structure to contain the RPF header information, plus a data structure to hold an indicator variable for each of the keyword fields, in addition to the function declarations for each of the functions defined in rpftools.c. For a 
Data Structure Definitions

\section{Reference Section Overview}

more detailed description of the keywords contained in the RPF header, see the document, RPF keyword data dictionary.

The RPFTools library, rpftools.o, is generated from the source code module, rpftools.c. It contains various ANSI C functions for reading, writing, and printing RPF data files. Its dependencies include the HDF include files (HDF inc directory) and libraries (HDF bin and lib directories), and the standard C libraries (include files and object modules). Non-HDF include files required at compilation time are rpftools. $h$, and clhtools. $h$. NonHDF object modules required at linking time are rpftools.o, and clhtools.o. The two files, clhtools.h and clhtools.o, are the include file and object module, respectively, for a collection of miscellaneous ANSI C utility functions used by RPFTools.

Example Usage. Use read_rpf_page to read a page of information from an RPF file. Use write_rpf_page to write a page of information to an RPF file.

This section of the RPFTools Reference Manual contains a listing of every function contained in RPFTools.c. The functions are ordered alphabetically according to their $\mathrm{C}$ function name. Each function is described in the following form:

\section{Function name}

Return_type function_name (type paramaeter1, type parameter2,... type parameter2)

Parameter1 Definition of the first parameter

Parameter2 Definition of the second parameter

$\cdots$

ParameterN Definition of the Nth parameter

Purpose Section containing a short description of the function.

Return value Section describing the return value, if any, and error conditions.

Description This section describes the function and any special circumstances surrounding the use of this function. It identifies any functions that must precede it and describes any known complications related to the use of it.

Example

This optional section consists of one or more examples of the use of the function. The examples are not complete programs and are intended for illustrative purposes only. For complete programs and further descriptions of the function, refer to the RPFTools User's Guide.

Functions

\section{add_rpf_comment}

void add_rpf_comment (header_type *rpfheader, header_status_type *rpfheader_status, $\operatorname{char} * \mathrm{t}$ ) 
rpfheader

rpfheader_status

$\mathrm{t}$

\section{Purpose}

Return Value

Description
OUT:The space to store the RPF header information

OUT:The space to store the RPF header status information

IN:The string to add to the comment field

Adds a string to the RPF header comment field.

None.

This function adds a string to the header comment field. If there is already an existing comment, a newline ("In") is added before the new comment is appended. The resulting string is null terminated (" 0 ") and the rpfheader status field, rpfheader_status->got_valid_one, is set to $\mathrm{ON}$ before the function returns.

\section{close_rpf_file}

void close_rpf_file (int32 *file_id, int32 sd_id, int32 sds_id, int32 vgroup_id)
file_id
IN:The RPF file id
sd_id
IN:The RPF data SD id
sds_id
IN:The RPF data SDS id
vgroup_id
IN:The RPF data vgroup id

Purpose

Return Value

Description
Terminates access to the SD interface and the V interface, and then closes an RPF file.

None.

This function terminates access to the SD interface designated by the sd_id and the sds_id, terminates access to the $\mathrm{V}$ interface designated by the vgroup_id, and then closes an RPF file designated by the file_id.

\section{free_rpf_header}

void free_rpf_header (header_type *rpfheader)

rpfheader

Purpose

Return Value

Description
IN: A pointer to the RPF data structure to destroy.

Frees the space used by the RPF header.

None.

This function frees the memory space previously allocated to hold the full RPF header information. Use this destructor function to avoid memory leaks due to incomplete release of header storage space. 


\section{get_line}

int get_line $($ char *s, FILE *fp)

s

fp

Purpose

Return Value

Description
OUT:The string of input data

IN:The pointer to the pre-opened file to be read

Get a single valid line of input data while ignoring comment lines.

It returns a 0 if it finds the EOF at the beginning of a line, a -1 if it finds a \# at the beginning of a line (comment line), and a 1 otherwise.

This function grabs a single valid line of input data while ignoring comment lines that are indicated by a leading \#. The original version was written for the UNIX operating system and obtained from Mark Wagner. During the most recent port to the Macintosh platform, it was rewritten. It now grabs a line as delimited by a newline $(\backslash n)$, a carriage return $(\backslash \mathrm{r})$ or an end of file (EOF).

\section{have_rpf_file}

void have_rpf_file (char *rpffname, int *eflag)

$\begin{array}{ll}\text { rpffname } & \text { IN:The name of the RPF file } \\ \text { eflag } & \text { OUT:An error flag, initialized to } 0 . \\ \text { Purpose } & \text { Determines whether a file is an RPF file. } \\ \text { Return Value } & \text { None. }\end{array}$

Description

This function determines whether the file specified by rpffname is indeed an RPF file.

Eflag is set to 1 if the file not found. It is set to 2 if the file that is found is not an RPF file.

\section{init_cdf_data}

specdat_type *init_cdf_data (int tsize)

tsize

Purpose

Return Value
IN:the initial number of bins in the data arrays Initialize the space to hold the CDF data.

A pointer to the initialized CDF data structure. 
Description

header_type *init_rpf_header (void)

Purpose

Return Value

Description

\section{init_rpf_header}

This function creates and then initializes the space to hold the CDF data.

If tsize is less than or equal to zero, tsize defaults to 42 . The data arrays, e_center, flux, d1, d2, and d3 are malloc-ed to a length of tsize and each value is set to 0.0 . The e_edge data array is malloc-ed to a dize of tsize +1 to accommodate the bin edges and then each value is set to 0.0 .

The parameters nbins and xstart are set to 0 . The parameter asize is set to tsize. 
void parse_cdf_header (char *fname, header_type *rpfheader, header_status_type *rpfheader_status, int verbose, int *eflag)

$\begin{array}{ll}\begin{array}{l}\text { fname } \\ \text { rpfheader }\end{array} & \text { OUT:Ame of the CDF file. } \\ \text { rpfheader_status } & \text { OUT:A pointer to the RPF header status information. } \\ \text { verbose } & \text { IN:A flag to control the amount of output. } \\ \text { eflag } & \text { OUT:An error flag, initialized to } 0 .\end{array}$

Purpose

Return Value

Description
Parses the header of a CDF file and fills the corresponding variales in a RPF header.

None.

This function opens an ASCII CDF file, parses its header line by line, places whatever information it can into a RPF header structure, and sets the corresponding variables in the RPF header status structure to $\mathrm{ON}$.

If the verbose flag is set to ON, when an RPF keyword is matched, it and its corresponding data value are printed to stdout. Also, when no RPF keyword is matched in the line of CDF header the message did not match rpf keyword:' followed by the CDF line is printed to stdout.

If the number_of_bins keyword value is redefined, a warning will be printed to stdout and eflag will be changed from its initial value of 0 to 1 . This can happen when the CDF keywords nchan and/or Channels= occurs more than once within the same CDF header section.

Note that the CDF file referred to here is the Common Data Format developed at LLNL and should not be confused with netCDF developed by UCAR.

This function covers only a small number of the possible keywords that can be found in a CDF file. Please see $A n$ Introduction to the Common Data Format (CDF) for further information for additional information.

\section{print_current_rpf_header}

void print_current_rpf_header (header_type *rpfheader, header_type *rpfheader_status)

$$
\begin{array}{ll}
\text { rpfheader } & \text { IN:A pointer to the space which stores the RPF header } \\
\text { information } & \\
\text { rpfheader_status } & \text { IN:A pointer to the space which stores the RPF header } \\
\text { status information }
\end{array}
$$


Purpose

Return Value

Description
Prints the portion of the RPF header that is currently valid.

None.

This function prints the fields of the RPF header data structure whose corresponding status variables are currently set to $\mathrm{ON}$ to stdout.

\section{print_full_rpf_header}

void print_full_rpf_header (header_type *rpfheader, header_type *rpfheader_status)

rpfheader IN:A pointer to the space which stores the RPF header information

rpfheader_status IN:A pointer to the space which stores the RPF header status information

Purpose

Prints the full RPF header.

Return Value

None.

Description

This function prints the full RPF header data structure to stdout.

\section{print_rpf_data}

void print_rpf_data (double *rpfdata, int32 rank, int32 *dims, int npl)

$\begin{array}{ll}\text { rpfdata } & \text { IN:The buffer containing the RPF data } \\ \text { rank } & \text { IN:The rank of the data block to be printed } \\ \text { dims } & \text { IN:Array specifying the size of each dimension } \\ \text { npl } & \text { IN:Number of data values to print per line } \\ \text { Purpose } & \text { Prints the RPF data. } \\ \text { Return Value } & \text { None. }\end{array}$

\section{Description}

This function prints the RPF data to stdout. The rank and dimension size of the data block is followed by the data itself. Currently, this function is set up for a rank of 2.

An example output looks as follows:

rank: 2

index: 0 dims[0]: 4

index: 1 dims[1]: 3

[0][0]: 2 [1][0]: 3 [2][0]: 24

[0][1]: $4 \quad[1][1]: 5 \quad[2][1]: 26$ 
[0][2]: $6 \quad[1][2]: 7 \quad[2][2]: 28$

[0][3]: 8 [1][3]: 0 [2][3]: 0

\section{readcdf}

void readcdf (char $*$ fname, int $*$ nbin, EF_TYPE $* *$ dat1, EF_TYPE $* *$ dat 2, EF_TYPE

$* *$ dat3, EF_TYPE **dat4, EF_TYPE **dat5, int max_size, int *eflag)

fname

nbin

dat1

dat2

dat3

dat4

dat5

max_size

eflag

Purpose

Return Value

Description
IN:The name of the CDF file to be opened, read, and then closed

OUT:The number of energy bins in the data matrix

OUT:A pointer to the energy bin data array

OUT:A pointer to the flux data array

OUT:A pointer to the relative error array

OUT:A pointer to the absolute error data array

OUT:A pointer to the original flux data array

IN:The maximum allowed size of the data array

OUT:An error flag, initialized to 0.

Read an ASCII CDF file containing up to five data values per line into separate double arrays.

None.

This function reads an ASCII CDF file containing from two to five data values per line into separate EF_TYPE arrays. The read continues is until an EOF is hit. Comments as indicated by a leading \# and blank lines are ignored.

The data arrays are resized as needed up to a maximum size of max_size elements. If the data to be returned occupies less than $75 \%$ of the malloc-ed space, the arrays are realloc-ed to use only the required space.

The parameter eflag is incremented each time it encounters a line of data with only one number. It is set to -1 if the ::ENDHEADER:: or ::endheader:: keyword is not found. It is set to -2 if there were no valid lines of data found.

\section{read_open_rpf_file}

void read_open_rpf_file (char *rpffname, int32*file_id, int32 sd_id, int *eflag)

rpffname IN:The name of the RPF file 

file_id
sd_id
eflag
Purpose
Return Value
Description
OUT:The RPF file id returned by HDF function Hopen
OUT:The RPF data SD id returned by HDF function SDstart
OUT:An error flag, initialized to 0 .
Opens an RPF file for reading.
None.
This function opens an RPF file for reading, initializes the SD interface, and initializes the RPF file for subse- quent vgroup access. The file_id and the sd_id are returned in the parameter list.
Eflag is set to 1 if the file not found. It is set to 2 if the file that is found is not an RPF file.

\section{read_rpf_data}

double *read_rpf_data (int32 vgroup_id, int32 sd_id, int32 *rank, int32 *dims, int *eflag)

vgroup_id
sd_id
rank
dims
eflag
Purpose
Return Value
Description

IN:The RPF vgroup id

IN:The RPF data SD id

OUT:The rank of the data to be read

OUT:Array specifying the size of each dimension

OUT:An error flag, initialized to 0 .

Reads the RPF data from an RPF data file.

Pointer to the array of RPF data values.

This function reads the RPF data section from a previously opened rpf file and returns a pointer to the buffer containing the RPF data. The rank and the dimension of the data is returned in the parameter list.

The space for dims needs to be allocated before entering this function. A safe way would be to define it as dims[MAX_VAR_DIMS]. MAX_VAR_DIMS is defined as 32 in the HDF include file hlimits.h.

Eflag is incremented by 1 for each read error that occurs from a call to SDreaddata.

\section{read_rpf_header}

void read_rpf_header (int32 file_id, int32 vgroup_id, header_type *rpfheader, header_status_type *rpfheader_status, int *eflag)

file_id IN:The RPF file id previously returned by HDF function Hopen. 


$\begin{array}{ll}\text { vgroup_id } & \text { IN: The id of the header vgroup to be read. } \\ \text { rpfheader } & \text { OUT:The RPF header information. } \\ \text { rpfheader_status } & \text { OUT:The RPF header status information. } \\ \text { eflag } & \text { OUT:An error flag, initialized to } 0 . \\ \text { Purpose } & \text { Reads an RPF header from an RPF data file. } \\ \text { Return Value } & \text { None. }\end{array}$

Description
This function reads the RPF header vgroup from a previously opened rpf file. It fills the RPF header data structure and sets the corresponding indicator variables in the header_status structure to "ON".

Eflag is incremented by 1 for each read error. It is incremented by 1000 for each time the vdata class and name does not match those for an RPF keyword.

\section{read_rpf_page}

void read_rpf_page (char *rpffname, int pageindex, header_type **rpfheader, header_status_type $* *$ rpfheader_status, double $* *$ rpfdata, int $32 *$ rank, int32*dims, int *eflag)

rpffname
pageindex
rpfheader
rpfheader_status
rpfdata
rank
dims
eflag

Purpose

Return Value

Description
IN:The name of the RPF file

IN:The index of the RPF page to read.

OUT: A pointer to the RPF header structure

OUT: A pointer to the RPF header status structure

OUT:A pointer to the buffer containing the RPF data

OUT:The rank of the data to be read.

OUT:Array specifying the size of each dimension in the data block.

OUT:An error flag, initialized to 0 .

Reads a RPF page.

\section{None}

This function opens a RPF file and reads a page of RPF data.

The space for dims, start, and edges need to be allocated before entering this function. A safe way would be to define dims as dims[MAX_VAR_DIMS].

MAX_VAR_DIMS is defined as 32 in the HDF include file hlimits.h.

Eflag is initialized to 0 . It is set to -1 if the RPF page designated by pagename that is created from the pagein- 
dex is not found and the function returns from there. Eflag is set to -2 if there were any errors during the reading of the header vgroup. Eflag is set to -3 if there were any errors during the reading of the data vgroup. It is set to -4 if errors occurred during the reading of both the header and data vgroups.

\section{reset_rpf_header}

void reset_rpf_header (header_type *rpfheader)

rpfheader

IN: The RPF data structure to reset.

Purpose

Resets the RPF header values to initialized values.

Return Value

None.

Description

This function resets the values for the full RPF header. Character string variables are initialized to just hold one 10 to show that they start off as empty strings. Integer variables are set to 0 . Double variables will be set to 0.0 . Pointers to doubles are initialized to a length of 1 and the value of the first array space is set 0.0. See the RPF Keyword Data Dictionary for a complete description of the RPF header fields.

\section{strchop}

void strchop (char *instring, char *outstring)
instring
IN:The original string
outst
OUT: The original string truncated to remove trailing
Purpose white spaces
Return Value
Remove the trailing white spaces in a string.
Description
None.
This function removes the trailing white spaces in a string. The resulting string is null terminated (' $\left.10^{\prime}\right)$.

\section{write_open_rpf_file}
void write_open_rpf_file (char *rpffname, int32*file_id, int32 sd_id, int *eflag)
rpffname IN:The name of the RPF file
file_id OUT:The RPF file id returned by HDF function Hopen
sd_id OUT:The RPF data SD id returned by HDF function SDstart
eflag OUT:An error flag, initialized to 0 .
Purpose Opens an RPF file for writing.


Return Value

Description
None.

This function opens an RPF file for writing. The SD interface is then initialized and the file is prepared for vgroup access.

Eflag is set to 1 if the file not found. A new RPF file is then created with read/write access. Eflag is set to 2 if the file that is found is not an RPF file. The function then returns.

\section{write_rpf_data}

int32 write_rpf_data (int32 file_id, int32 sd_id, char *datasetname, double *rpfdata, int32 rank, int32*dims, int32*start, int32*edges, int *eflag)

file_id

sd_id

datasetname

rpfdata

rank

dims

start

edges

eflag

Purpose

Return Value

Description
IN:The RPF file id returned by HDF function Hopen

IN:The RPF data SD id returned by HDF function SDstart

IN:The name of the RPF data vgroup.

IN:The buffer containing the RPF data.

IN:The rank of the data to be written.

IN:Array specifying the size of dimension.

IN:Array specifying the starting location.

IN:Array specifying the number of values to be written along each dimension.

OUT:An error flag, initialized to 0 .

Writes the RPF data into an RPF data file.

The reference number of the RPF data SDS vgroup_id.

This function opens a RPF file, and writes the RPF data as an HDF scientific data set (SDS). It is up to the user to create the rpfdata block to pass to this function.

For example, Type $2 \mathrm{CDF}$ data storing a single gamma ray spectrum can be written as a rank 2 data matrix in which the 0th dimension, dims[0], is the number of bin edges (equivalent to the number of bins +1 ), and the 1 st dimension, dims[1], is equal to 3 . This would facilitate the storage of energy bin edge values, energy bin center values and bin fluxes.

Eflag is set to the number of errors that occurred during the writing of the data. 


\section{write_rpf_header}

int32 write_rpf_header (int32 file_id, char *headername, header_type*rpfheader, header_status_type *rpfheader_status, int *eflag)

file_id

headername

rpfheader

rpfheader_status

eflag

Purpose

Return Value

Description
IN:The RPF file id previously returned by HDF function Hopen

IN:The name of the RPF header vgroup.

IN:The space which stores the RPF header information.

IN:The space which stores the RPF header status information.

OUT:An error flag, initialized to 0.

Writes an RPF header into an RPF data file.

The vgroup_id of the header vgroup that is written

This function writes the RPF header information into a vgroup of a previously opened rpf file designated by its file_id. Only those keywords whose corresponding rpfheader_status fields are set to ON will be written.

Eflag is set to the number of errors that occurred during the writing of the header data using VSwrite.

\section{write_rpf_page}

void write_rpf_page (char *rpffname, int pageindex, header_type **rpfheader, header_status_type **rpfheader_status, double **rpfdata, int32 *rank, int32*dims, int32*start, int32*edges, int *eflag)

rpffname
pageindex
Rpfheader
rpfheader_status
rpfdata
rank
dims
start
edges
eflag

Purpose
IN:The name of the RPF file.

IN:The index of the RPF page to return.

OUT: A pointer to the pointer to the RPF header structure.

OUT: A pointer to the pointer to the RPF header status structure.

OUT:A pointer to the buffer containing the RPF data.

OUT:The rank of the data to be written.

OUT:Array specifying the size of dimension.

OUT:Array specifying the starting location.

OUT:Array specifying the number of values to be written along each dimension.

OUT:An error flag, initialized to 0 .

Writes a RPF page. 
Return Value

Description
None.

This function opens a RPF file and writes a page of RPF data.

Eflag is initialized to 0 . It is set to -1 if the file designated by rpffname is found not to be an RPF file and the function returns from there. Eflag is set to -2 if there were any errors during the writing of the header vgroup. Eflag is set to -3 if there were any errors during the writing of the data vgroup. It is set to -4 if errors occurred during the writing of both the header and data vgroups. 


\section{Data Dictionary: Header Keywords for the Radiation Physics Format}

\section{Description}

\section{Adding/modifying keywords}

It is imperative for the portability of data between our applications to have a standard set of keywords in the header information section(s) of our RPF files. This section contains the data dictionary for the keywords that are used to describe the fields in our RPF files. The initial list of keywords was declared by fiat by the RPF file designer, Cheri Ham (ham1@1lnl.gov) after receiving input from users Tom Gosnell (gosnell1@llnl.gov), Zach Koenig (koenig@1lnl.gov), Bert Pohl (pohl1@1lnl.gov), Dave Knapp (knapp2@1lnl.gov), Jim Wolford (wolford@llnl.gov), and Alexis Schach von Wittenau (schachvonwittenau@llnl.gov).

In order for the RPF spectral file header format and its associated software to be flexible and yet maintain their extensibility with minimal backtracking and reworking, the definitions of the header keywords and their uses must be strictly controlled. It is expected that the list of header keywords will evolve with use over time. Procedures to add, modify, delete, and exclude header keywords are described below. These procedures, although draconian in appearance, must be strictly followed until or unless they are formally modified. Maintaining a tight rein on the keywords from the beginning will lessen the chaos that keyword changes will cause in the future.

Remember, not all keywords will appear in each RPF header. Only those keywords relevant to each particular header will be included in the RPF file.

Changes and additions may be made to the accepted keyword list according to the following 6-step procedure.

Step 1: Submit written request for data dictionary modification to the person in charge of the keyword data dictionary. Initially and currently the keyword Czar will be the RPF file designer, Cheri Ham. For modifications of existing keywords, this request should include the a list of the current keyword(s) to be modified, a list of the corresponding keyword(s) that the requester would like to be used, and a justification for each of the requested changes. To incorporate new keywords, the request should include a list of the new keyword(s) to be added, a complete definition of each of the new keywords, and justification for each of the requested new keywords.

Step 2: The keyword Czar will submit the written request to the appropriate subset of the local community of RPF file users for feedback. They will have 7 calendar days to provide oral and/or written feedback. Initially and currently, the members of the keyword review committee will be Tom Gosnell (gosnell1 @llnl.gov), Zach Koenig (koenig@1lnl.gov), Bert Pohl (pohl1@1lnl.gov), Dave Knapp (knapp2@1lnl.gov), Jim Wolford (wolford@llnl.gov), and Alexis Schach von Wittenau (schachvonwittenau@1lnl.gov).

Step 3: The keyword Czar will consider the initial request and all the feedback, resolve any and all conflicts, and render a ruling upon the request, plus generate the required update to the RPF spectral file accepted keyword data dictionary. 
Step 4. A detailed written ruling on the request to add/modify keywords will be disseminated to the local RPF file user community. This will include information on the original request, the feedback received, and the ruling rendered, plus any other relevant information such as the potential keyword data dictionary entry. The local user community will have 7 calendar days to submit a written appeal to the keyword Czar.

Step 5. They keyword Czar will resolve any appeals by repeating the described process as required.

Step 6. They keyword Czar will make a detailed entry into the keyword data dictionary logbook and update the keyword data dictionary as required.

Deleting/excluding keywords

Modifying the keyword change procedure
There will be occasions that certain keywords and the information they represent will be deleted or excluded from the accepted list of keywords. The following describes the procedure to delete or exclude a keyword from the accepted header keyword list.

Step 1: Submit written request for data dictionary modification to the person in charge of the keyword data dictionary. Initially and currently the keyword Czar will be the RPF file designer, Cheri Ham. This request should include the a list of the current keyword(s) to be deleted or excluded and a justification for each of the requested changes.

Step 2: The keyword Czar will submit the written request to the appropriate subset of the local community of RPF file users for feedback. They will have 7 calendar days to provide oral and/or written feedback. Initially and currently, the members of the keyword review committee will be Tom Gosnell (gosnell1 @ llnl.gov), Zach Koenig (koenig@llnl.gov), Bert Pohl (pohl1@llnl.gov), Dave Knapp (knapp2@llnl.gov), Jim Wolford (wolford@llnl.gov), and Alexis Schach von Wittenau (schachvonwittenau@1lnl.gov).

Step 3: The keyword Czar will consider the initial request and all the feedback, resolve any and all conflicts, and render a ruling upon the request, plus generate the required update to the RPF spectral file rejected keyword data dictionary.

Step 4. A detailed written ruling on the request to delete/exclude keywords will be disseminated to the local RPF file user community. This will include information on the original request, the feedback received, and the ruling rendered, plus any other relevant information. The local user community will have 7 calendar days to submit a written appeal to the keyword Czar.

Step 5. They keyword Czar will resolve any appeals by repeating the described process as required.

Step 6. They keyword Czar will make a detailed entry into the keyword data dictionary logbook and update the keyword data dictionary as required.

It its allowed that the procedures for changing the header keyword list might require modification at some point in time. 
Step 1: Submit written request for procedure modification to the person in charge of the keyword data dictionary. Initially and currently the keyword Czar will be the RPF file designer, Cheri Ham. The requester should include a description of and a justification for each of the requested changes, additions, and/or deletions.

Step 2: The keyword Czar will consider the request, and if deemed necessary will submit the written request to the appropriate subset of the local community of RPF file users for feedback. They will have 7 calendar days to provide oral and/or written feedback. Initially and currently, the members of the keyword review committee will be Tom Gosnell (gosnell1@1lnl.gov), Zach Koenig (koenig@1lnl.gov), Bert Pohl (poh11@1lnl.gov), Dave Knapp (knapp2@1lnl.gov), Jim Wolford (wolford@1lnl.gov), and Alexis Schach von Wittenau (schachvonwittenau@ @llnl.gov).

Step 3: The keyword Czar will consider the initial request and all the feedback, resolve any and all conflicts, and render a ruling upon the request, plus generate the required update to the Modifying the keyword change procedure.

Step 4. A detailed written ruling on the request to add/modify keywords will be disseminated to the local RPF file user community. This will include information on the original request, the feedback received, and the ruling rendered, plus any other relevant information. The local user community will have 7 calendar days to submit a written appeal to the keyword Czar.

Step 5. They keyword Czar will resolve any appeals by repeating the described process as required.

Step 6. They keyword Czar will make a detailed entry into the keyword data dictionary logbook and update the keyword change procedure as required. 
Data Dictionary: Header Keywords for the Radiation Physics Format

RPF spectral file accepted keyword data dictionary
This data dictionary contains a list of the accepted HDF spectral file keywords, their defined data type, an example, and a detailed description of the information that they contain. This list is ordered to group keywords by functionality as opposed to alphabetically.

\section{File creation keywords}

The following three keywords preserve information on the initial creation of the HDF spectral data file.

$\begin{array}{lll}\text { date_file_written } & \text { char } * & \begin{array}{l}\text { Ex: mm-dd-yyyy e.g., 03-14-1995. This ASCII string contains the original date } \\ \text { that this file was written. }\end{array} \\ \text { time_file_written } & \text { char } * & \begin{array}{l}\text { Ex: hh:mm:ss XXX e.g., 14:12:00 PST. This ASCII string contains the original } \\ \text { time that this file was written. }\end{array} \\ \text { original_filename } & \text { char } * & \begin{array}{l}\text { E.g., IdatalU071_414.CNF. This ASCII string contains the original file name } \\ \text { used when this file was written. }\end{array}\end{array}$

\section{Experiment identification keywords}

The following keywords contain basic information to identify the experiment.

$\begin{array}{lll}\begin{array}{l}\text { location } \\ \text { experimenters_names }\end{array} & \text { char } * & \begin{array}{l}\text { This ASCII string contains the location where the experiment was performed. } \\ \text { This ASCII string contains the names of the people involved in conducting the } \\ \text { experiment. }\end{array} \\ \text { experiment_id } & \text { char } * & \begin{array}{l}\text { This ASCII string contains the experiment identification label. } \\ \text { run_number }\end{array} \\ \text { number_of_channels } & \text { int32 } & \begin{array}{l}\text { This is the run number which resulted in the data attached to this header. } \\ \text { This contains the number of channels in the data block associated with this } \\ \text { header. }\end{array}\end{array}$


Data Dictionary: Header Keywords for the Radiation Physics Format

\section{Hardware Identification Keywords}

These keywords identify the experimental hardware and their parameters. The use of high_voltage, fine_gain, coarse_gain, and shaping_time are dependent upon the type of instrument used.

$\begin{array}{lll}\text { instrument_type } & \text { char } * & \text { This ASCII string describes the type of instrument used in the experiment. } \\ \text { instrument_id } & \text { char } * & \text { This ASCII string contains the instrument serial number or other identification. } \\ \text { high_voltage } & \text { real64 } & \text { This describes the high voltage adjustment used. } \\ \text { fine_gain } & \text { real64 } & \text { This describes the fine gain adjustment used. } \\ \text { coarse_gain } & \text { real64 } & \text { This describes the coarse gain adjustment used. } \\ \text { shaping_time } & \text { real64 } & \text { This describes the shaping time used. } \\ \text { detector_type } & \text { char } * & \text { This ASCII string describes the type of detector used in the experiment. } \\ \text { detector_id } & \text { char } * & \text { This ASCII string contains the detector serial number or other identification. }\end{array}$

\section{Experiment Time Keywords}

The following keywords contain timing and location information for the experiment.

$\begin{array}{lll}\text { count_clock_time } & \text { char } * & \begin{array}{l}\text { Ex: hh:mm:ss XXX e.g., 14:12:00 PST. This ASCII string contains the clock time } \\ \text { when the experiment was started stored in ANSI standard format. }\end{array} \\ \text { count_start_date } & \text { char } * & \begin{array}{l}\text { Ex: mm-dd-yyy e.g., 03-14-1995. This ASCII string contains the date when the } \\ \text { experiment's data collection was started stored in ANSI standard format. }\end{array} \\ \text { count_start_time } & \text { char } * & \begin{array}{l}\text { Ex: hh:mm:ss XXX e.g., 14:12:00 PST. This ASCII string contains the time when } \\ \text { the experiment's data collection was started stored in ANSI standard format. }\end{array} \\ \text { count_live_time } & \text { real64 } & \text { This contains the live time of the experiment measured in decimal seconds. } \\ \text { count_dead_time } & \text { real64 } & \text { This contains the dead time of the experiment measured in decimal seconds. }\end{array}$


Data Dictionary: Header Keywords for the Radiation Physics Format

\section{Energy Calibration Keywords}

These keywords describe various energy calibration parameters.

energy_calibration_type

intercept

slope

quad

number_of_energy_coeffs

energy_calibration_coeffs

number_of_energy_bin_edges char* This describes the type of energy calibration used. Valid options are none, normal, polynomial, and binned. None means that no calibration is available.

Normal uses the keywords intercept, slope, and quadratic to calculate the energy calibration via the formula

energy value $=[$ quadratic $*$ bin_number $*$ bin_number $]+[$ slope $*$ bin_number $]+[$ offset $]$. Polynomial uses the number_of_energy_coeffs coefficients stored in energy_calibration_coeffs to calculate the energy calibration using a higher order polynomial equation. The intercept is stored in energy_calibration_coeffs[0].

The slope is stored in energy_calibration_coeffs[1]. The quadratic term is stored in energy_calibration_coeffs[2], and so on. The spectrum option stores the number_of_energy_bin_edges bin edges in the energy_calibration_spectrum array.

real64 This is the intercept for the normal mode of energy calibration.

real64 This is the slope for the normal mode of energy calibration.

real64 This is the quadratic factor for the normal mode of energy calibration. int16

real64 array
This is the number of energy coefficients for the polynomial calibration option.

This contains the actual coefficients for the polynomial calibration option. The intercept is stored in energy_calibration_coeffs[0]. The slope is stored in energy_calibration_coeffs[1]. The quadratic term is stored in energy_calibration_coeffs[2], and so on.

This is the number of energy bin edges required for the energy calibration. This value should equal [number_of_channels + 1]. 
Data Dictionary: Header Keywords for the Radiation Physics Format

$\begin{array}{ll}\text { energy_calibration_spectrum } \quad \text { real64 array } & \begin{array}{l}\text { This contains the energy bin edges for the data associated with this header. } \\ \text { energy_calibration_spectrum[0] contains the leftmost bin edge. The remaining } \\ \text { [number_of_energy_bin_edges - 1] bin edges are the energies of the right bin } \\ \text { edges. }\end{array} \\ \text { energy_calibration_filename } \quad \text { char } * & \begin{array}{l}\text { This ASCII string contains the name of the file from which energy calibration } \\ \text { information was obtained for the data associated with this header. }\end{array}\end{array}$

\section{Efficiency calibration keywords}

These keywords describe various peak efficiency calibration parameters.

efficiency_calibration_type char $* \quad$ This describes the type of efficiency calibration used. Valid options are none, parameter, and spectrum. None means that no calibration is available. Parameter uses the number_of_efficiency_coeffs parameters stored in efficiency_calibration_params to store the efficiency calibration information. The spectrum option stores the efficiency calibration information in the efficiency_calibration_spectrum array.

efficiency_calibration_filename_char* This ASCII string contains the name of the file from which energy calibration information was obtained for the data associated with this header

\section{Geometry keywords}

These keywords describe the geometry of the experiment. $\begin{array}{ll}\text { source_detector_distance } & \text { real64 } \\ \text { This is the closest approach [a.k.a. fact-to-face] distance from the source to the } \\ \text { detectors. It is assumed to be the closest approach distance unless the }\end{array}$ geometry_description field describes otherwise.

geometry_description char * This describes relevant experimental geometry information.

\%_solid_angle real64 This is the \% solid angle. 
Data Dictionary: Header Keywords for the Radiation Physics Format

\section{Material description keywords}

These keywords describe the material(s) used in the experiment and are grouped according to source, absorber, collimator, and shield parameters that are described in more detail below.

\section{Source Description Keywords}

These keywords describe the source used in the experiment.

$\begin{array}{lll}\text { source_description } & \text { char } * & \text { This describes the source used in the experiment. } \\ \text { source_id } & \text { char } * & \text { This is the serial number or other identifier for the source used in the experiment. } \\ \text { source_material } & \text { char } * & \text { This is the index of the source material. } \\ \text { declared_enrichment } & \text { real64 } & \text { This is the declared enrichment value. } \\ \text { declared_enrichment_error } & \text { real64 } & \text { This is the error associated with the declared enrichment value. } \\ \text { measured_enrichment } & \text { real64 } & \text { This is the measured enrichment value. } \\ \text { measured_enrichment_error } & \text { real64 } & \text { This is the error associated with the measured enrichment value. } \\ \text { wall_thickness } & \text { real64 } & \text { This is the wall thickness value. } \\ \text { thickness_error } & \text { real64 } & \text { This is the error associated with the wall thickness value. } \\ \text { geom_correction } & \text { real64 } & \text { This is the geometric correction factor associated with this source. } \\ \text { wall_material } & \text { real64 } & \text { This is the index of the wall material. }\end{array}$

\section{Absorber description keywords}

These keywords describe the absorber(s) used in the experiment.

number_of_absorbers

int32

This is the number of absorber layers which are described in the absorber data block.

absorber_material

char* array

This is the description of the absorber material. 
Data Dictionary: Header Keywords for the Radiation Physics Format

absorber_thickness real64 array This is the thickness of the absorber material layer.

\section{Collimator description keywords}

These keywords describe the collimator(s) used in the experiment

number_of_collimators int32 This is the number of collimators which are described in the collimator data block.

collimator_type char*array Currently acceptable collimator types include none, cylindrical, and complex

collimator_description char*array This describes the collimators

\section{Shield Description keywords}

These keywords describe the shielding material(s) used in the experiment.

$\begin{array}{lll}\text { number_of_shields } & \text { int32 } & \text { This is the number of shields that are described in the shields data block } \\ \text { shield_type } & \text { char *array } & \text { This describes the shield type } \\ \text { shield_description } & \text { char *array } & \text { This describes the shields }\end{array}$

\section{Comment keywords}

This keyword field allows for user comments.

comments char *

This is an ASCII string that can include newlines, tabs, etc., in which the user may make additional comments. It is terminated with a null character ('In'). 
Data Dictionary: Header Keywords for the Radiation Physics Format

HDF spectral file rejected keyword data dictionary
There will be occasions that certain keywords and the information they represent will be excluded from the accepted list of keywords. This data dictionary contains a list of the rejected HDF spectral file keywords, their description of the information, and why they were rejected.

count_end_time
It was decided by consensus by the attendees at the LDRD meeting on 12/15 (who also happened to be the initial members of the keyword review committee will be Tom Gosnell

(gosnell1@1lnl.gov), Zach Koenig (koenig@llnl.gov), Bert Pohl (pohl1@llnl.gov), Dave Knapp (knapp2@1lnl.gov), Jim Wolford (wolford@1lnl.gov), and Alexis Schach von Wittenau (schachvonwittenau@llnl.gov) that this field was not necessary since the information that it would have contained can be calculated from other header fields. 


\section{RPF Future Directions}

Current Status of RPF

\author{
RPF and Event-Mode \\ Data
}

Single Experiment, Multiple Detectors
As mentioned in the introduction, the RPFTools subroutine library was frozen at version 4.1r3 of HDF. Since that time NCSA has released version 5 of HDF and, if the RPFTools are to be applied to a significant data storage application, it would be wise to upgrade them to be compatible with the latest version of HDF. Because the entire RPFTools library source code comprises only 4500 lines, many of them comment lines, this upgrade would not be arduous.

It is sometimes desirable to collect nuclear data in event mode. Event mode data are a temporal series of information vectors associated with each detected event such as detector ID, pulse height address, arrival time, GPS coordinates, altitude, temperature, humidity, barometric pressure, etc.

Currently the Radiation Physics Format does not accommodate event-mode data. However, the extensible nature of the RPF means that event mode data can be stored with ease. Since RPF files are self-describing, a variety of event vectors can be accommodated. Should we wish to extend the RPF to include event-mode data, the event vectors will be buffered and then stored to the RPF file as slabs in order to increase data collection throughput.

RPFTools functionality currently supports writing more than one data page per file. The stand-alone RPF application cdf2rpf demonstrates this when translating a CDF file which contains a two-part spectrum. The photopeak spectrum and the continuum spectrum are detected by a decrease in the list of bin energies that should be monotonically increasing for a spectrum. It then writes the data into one RPF file as two separate data pages with the same information in the header.

One of the strengths of the RPF file structure lies in its ability to group related data. The challenge lies in determining what is related and what constitutes a group and therefore should be contained in one RPF file. This is dependent upon not only what is being measured but at what level the grouping is to be done.

For example, on a very high level, all the data related to a particular experiment could be considered a group and therefore be written to a single RPF file. But on closer inspection, there could be several different detector types (e.g. NaI, HPGe, neutron, plastic scintillator, temperature, barometric pressure, etc.) and perhaps each detector type should be a group and therefore an RPF file. However, it may make more sense if groups were based upon location and an RPF file would contain data from all co-located detectors.

Determining the optimal grouping of data inside an RPF file must take into consideration not only the type of data that are to be stored but also the eventual use of those data. Ideally, this should be done in a thoughtful way before the data are gathered.

To make the RPF viable for long-term use, a rigid structure must be imposed upon a particular implementation of the RPF data files. Remember that although the RPF files are self describing, the data and tools to manipulate them are very tightly coupled. This means that it must be decided a priori which data will be stored and what keywords will describe 
Using RPFTools In a Large-Scale, MultipleDetector Experiment it for each detector, experiment, etc., and that any additions or modifications of the RPF structure must be done according to a strict protocol.

This is not as daunting a task as it may seem upon first inspection. It has been successfully done for CDF files. The standalone programs, cdf2rpf and showrpf are examples of that.

The RPF was intended to be adapted to accommodate the copious amounts of data that result from a large-scale, multiple-detector experiment. It just requires a bit of thoughtful planning beforehand to decide which data need to be included and how they should be partitioned. Then the appropriate programs can be written using the RPFTools library to store, retrieve, and manipulate the resulting RPF files.

An RPF data file consists of one or more pages of information. A page consists of a header describing the page plus its associated data. Each page is stored as an HDF vgroup.

One scheme establishes the RPF internal vgroup hierarchy with the overall experiment at the root of the tree. The next level of interior nodes contains portions of the experiment broken into temporal chunks. This could segregate the members into chunks collected daily, in the morning, or in the afternoon. The members of these temporal vgroups contain portions of the experiment broken into spatial chunks. This would group data from colocated detectors. The leaf nodes of this simple hierarchical tree are data pages describing each detector.

Headers must be established that reflect relevant information at each node level. For example, the header of the root page should contain information describing the overall experiment such as experiment name, date, location, experimenter names, sponsoring organization, etc. The first interior node level should contain information concerning the overall start and stop times of the portion of the experiment described in its members. The second interior node level should contain information concerning the location (e.g. GPS co-ordinates) of the detectors described in its members.

Experimental setup, source location, and other header information may be attached at various levels. It needs to be decided beforehand where it makes most sense.

Currently we have a set of keywords to describe gamma-ray spectra. Sets of keywords to describe neutron, temperature, barometric pressure, and other data need to be developed. It is expected that the neutron keywords will be similar to the gamma keywords. The temperature and barometric keyword sets should be simpler.

Currently we have an established vdata to describe gamma-ray spectra. It contains energy bin edges, energy bin centers, and flux values to describe a spectrum. A vdata for neutron data should include a temporal index and the count value. The vdatas for temperature and barometric pressure would contain a temporal value and the temperature or pressure, respectively.

The data label for the gamma-ray spectra is gamma_data. Data labels for the other types of data mentioned would be neutron_data, temperature, and barometric_pressure. 


\section{Appendix A: An Introduction to the Common Data Format (CDF)}

Anatomy of a CDF file

\section{Example CDF file}

The Common Data Format (CDF) was developed in the mid-1980's as a means to store gamma ray spectral data in a manner which would be versatile in both the types and amounts of data to store, easily readable by a human user, and transportable across various computing platforms. The CDF spectral file format is an ASCII format in which can contain more than one page of data. A page of data consists of a header and its associated spectral data.

The header is delimited by $::$ beginheader $::$ and $::$ endheader $::$ (or $:: B E G I N H E A D E R::$ and $:: E N D H E A D E R::$, if you really must). Between these delimiting keywords are a user determined number of keyword \& value pairs, one pair per line. For example,

\section{FILETYPENO=2}

The FILETYPENO keyword designates the number of columns in the data section. For backwards/sideways compatibility please be sure to include this in future CDF files. Another keyword to include would be CHANNELS=.

The data consist of up to five space-delimited fields of data per line. Type 2 CDF files consist of energy bin edge, flux pairs that appear one pair per line. The first data pair is the leftmost energy bin edge with a dummy flux. The remaining pairs are the right energy bin edge with the flux of that bin. Type 5 CDF files are constructed in the same manner as the Type $2 \mathrm{CDF}$ files with the additional space delimited fields of relative error, absolute error, and original flux on each line.

There can be two-part data: peak data followed by continuum data. This is recognized by a decrease in the normally monotonically increasing energy values.

The CDF file is automatically recognized based upon the presence of the initial keyword $\therefore$ beginheader $:$ or $:: B E G I N H E A D E R::$.

An example CDF file follows. Not all required keywords are present (e.g. FILETYPENO). Also note that Dave Knapp had decided to preface his keywords with an ' $*$ '. This is not wrong, just different.

::beginheader::

TITLE=a_file_with_no_name

CHANNELS $=\quad 10$

TIMECAL= 03-01-96 09:59:46

slope....: $3.09249 \mathrm{E}+00$

quad.....: 0.00000E+00

offset...:-4.24517E+01 


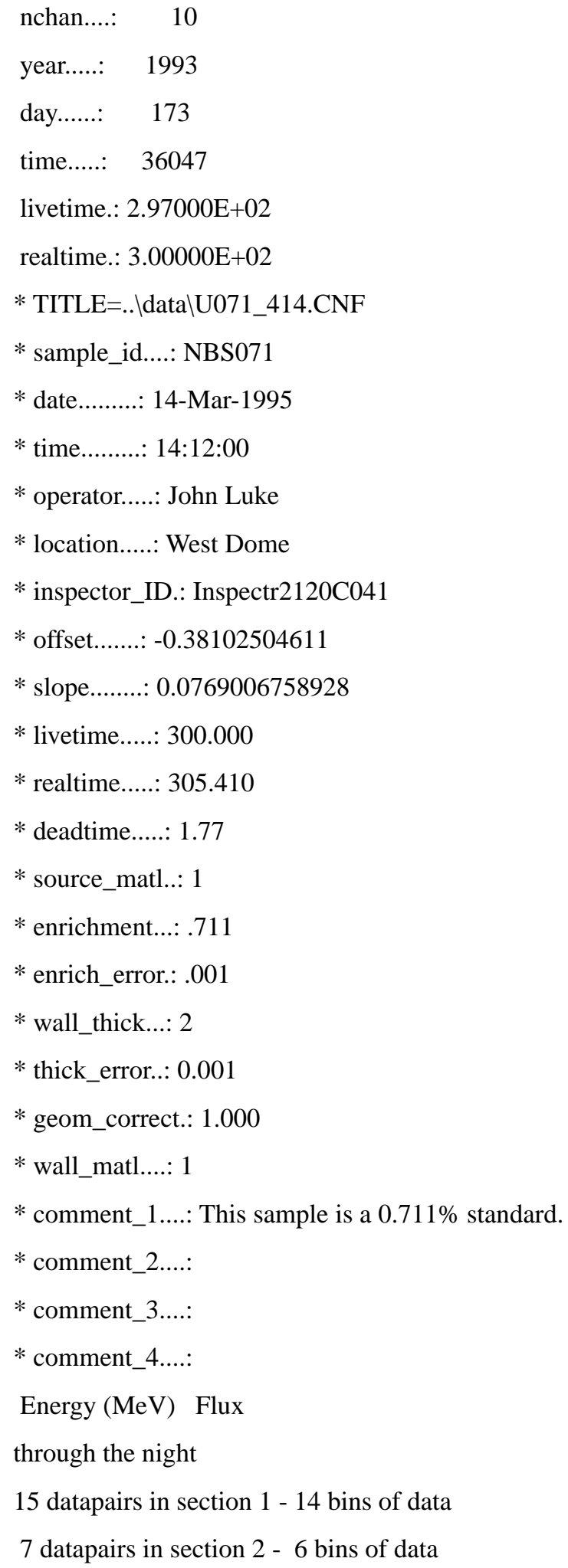


::ENDHEADER::

$-3.93593 \mathrm{E}-02 \quad 0.00000 \mathrm{E}+00$

142

344

546

748

950

1162

1364

1566

1768

Sample $\mathrm{C}$ code for reading \& writing CDF files
The following $\mathrm{C}$ code for reading and writing CDF files was stripped out of code written for Palatyi. No attampts were made here to streamline it for simple reading and writing functionality, or to undo the wonderful mung-job that Microsoft Word did on the original formatting. However, it should be fairly straightforward to do so.

read_cdf_file stripped out of getdata.c

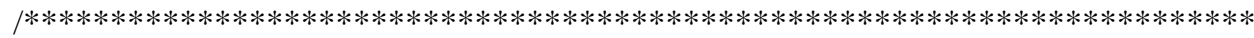

* read_cdf_file - read the cdf file

* 19 aug 1992 clh create

* 8 sep 1993 clh implement error handling if can't find ::ENDHEADER:: flag.

* 18 mar 1994 clh incorporate processing for two part cdf file into here.

* This has been tested and now works just fine.

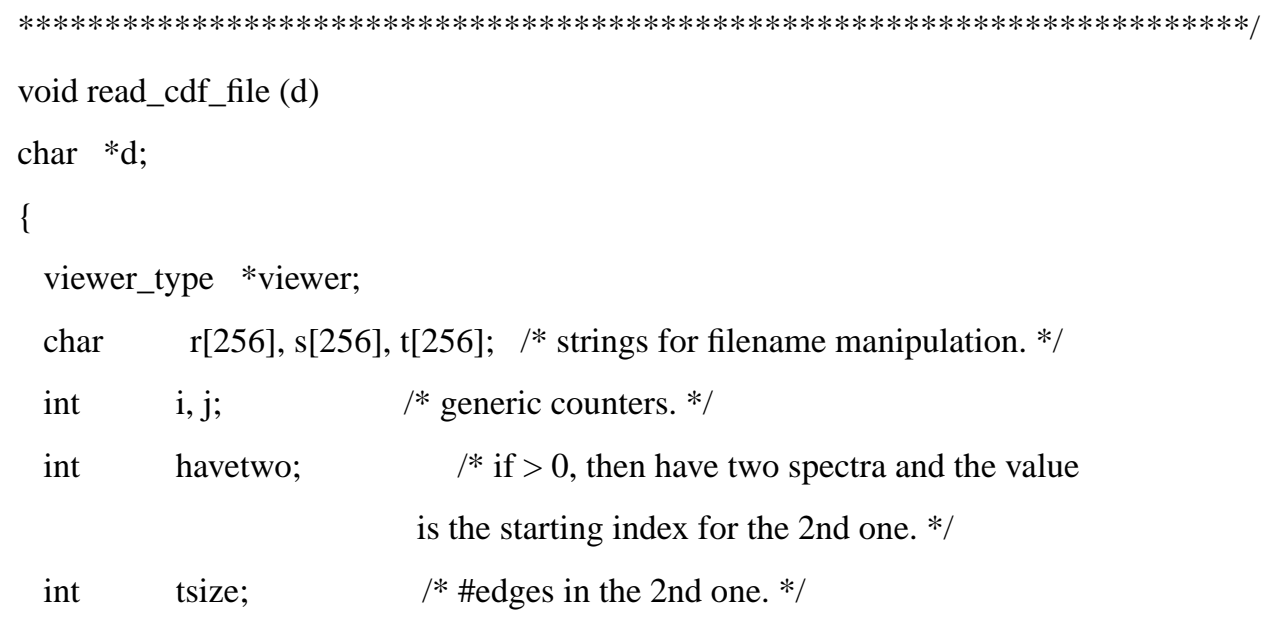




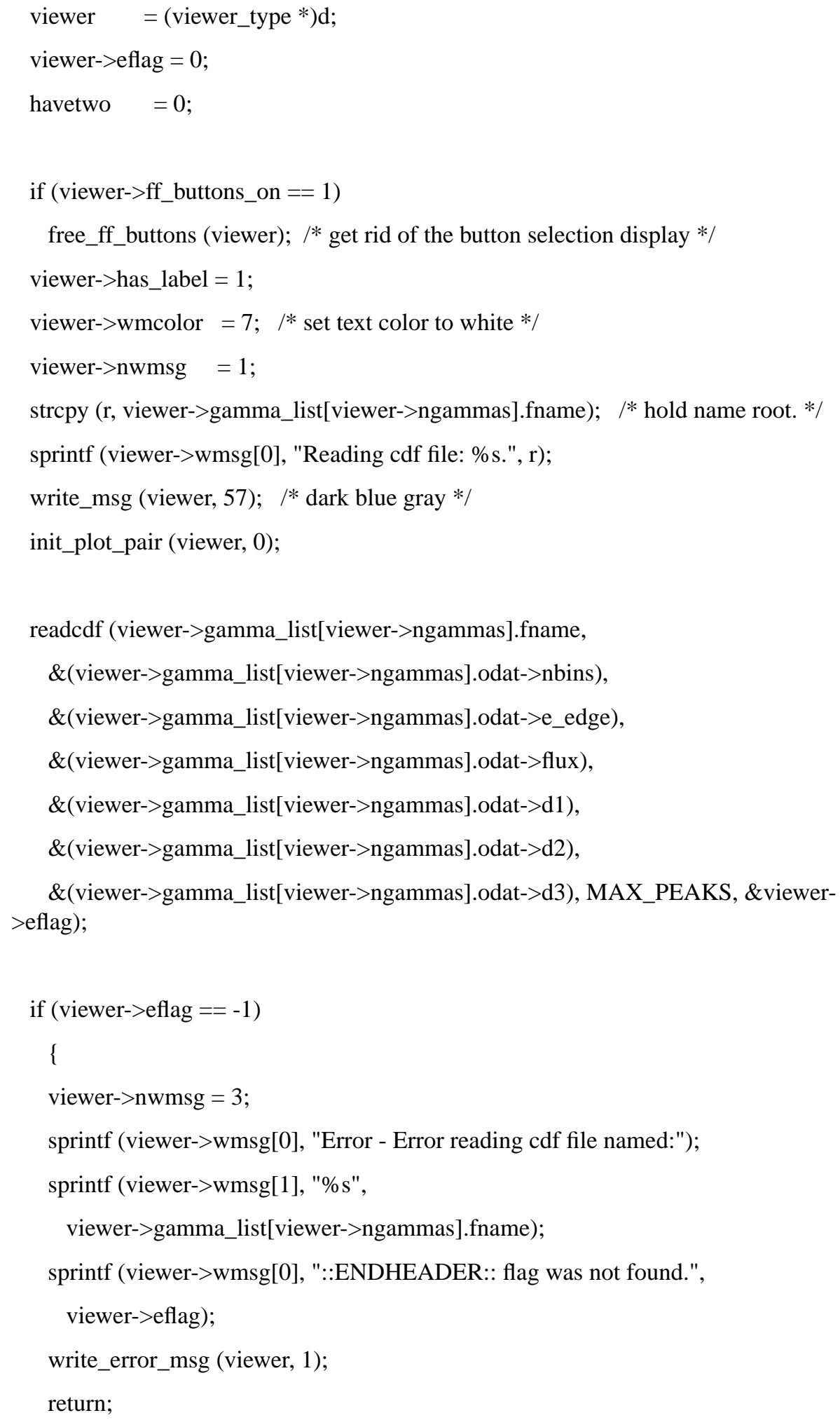




\section{Appendix A: An Introduction to the Common Data Format (CDF)}

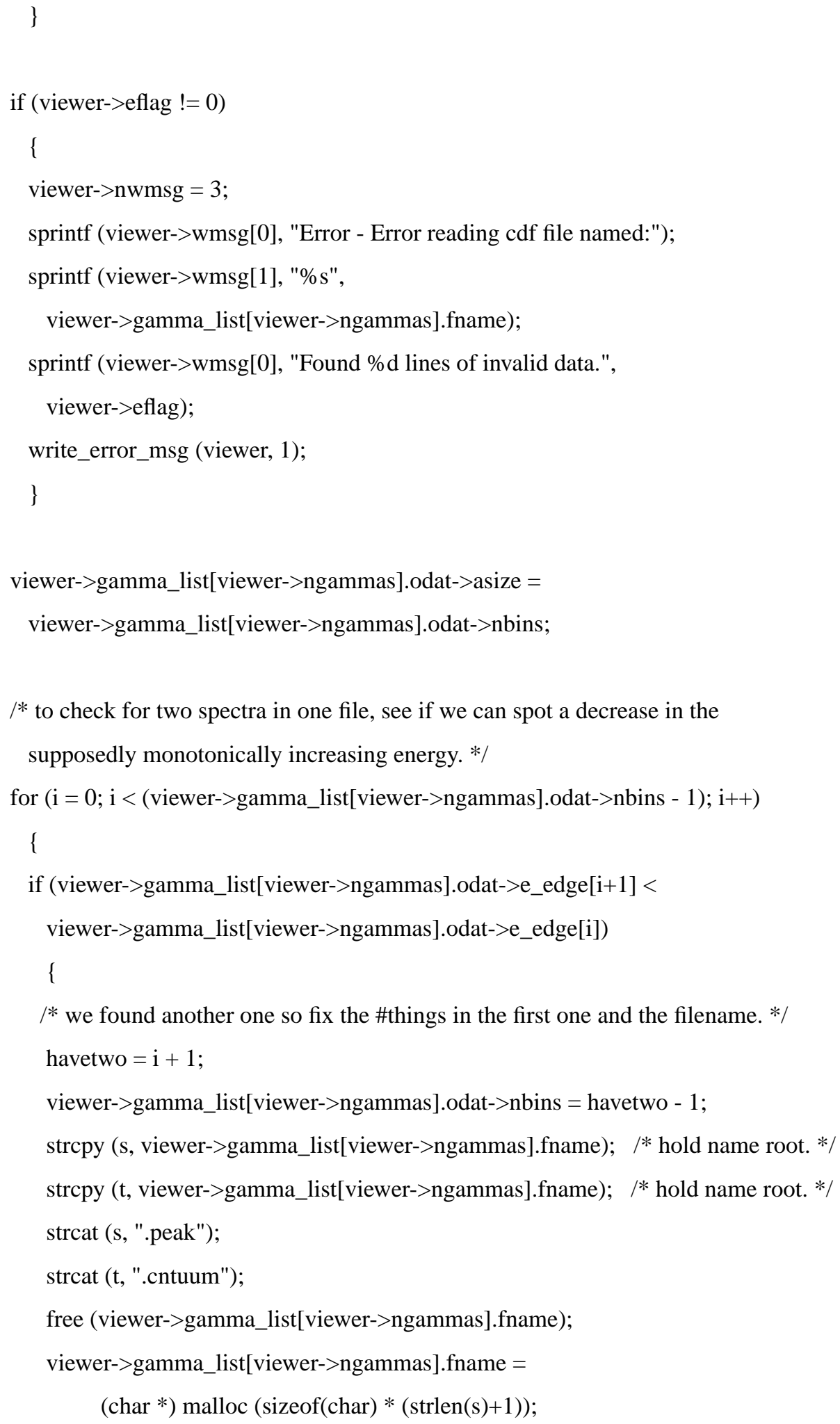




\section{Appendix A: An Introduction to the Common Data Format (CDF)}

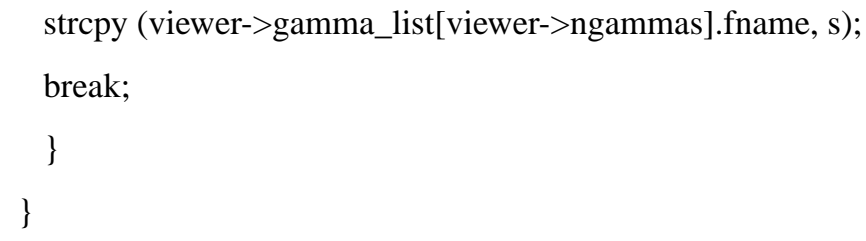

\section{\#undef DEBUG}

\#ifdef DEBUG

print_struct_specdat_type (viewer->gamma_list[viewer->ngammas].odat, 1); \#endif

/* set the other parameters for the first one.*/

viewer->gamma_list[viewer->ngammas].fmt_id = gdf_cdf;

viewer->gamma_list[viewer->ngammas].visible $=1$;

viewer->gamma_list[viewer->ngammas].color = return_next(MAX_WAVE_COLORS, viewer->used_colors);

viewer->used_colors[viewer->gamma_list[viewer->ngammas].color]++;

viewer->nwmsg $=1$;

sprintf (viewer->wmsg[0], "Finished reading CDF file: \%s.", r);

write_msg (viewer, 57); /* dark blue gray */ 
viewer->ngammas++; $/ *$ so we don't step on what we just made. */

$/ *$ if we have a second one make space for the 2 nd spectrum and fill it. */

if (havetwo >0) 


\begin{abstract}
viewer->gamma_list[viewer->ngammas].odat->nbins, \&viewer->gamma_list[viewer->ngammas].odat->flmin, \&viewer->gamma_list[viewer->ngammas].odat->flmax);
\end{abstract}

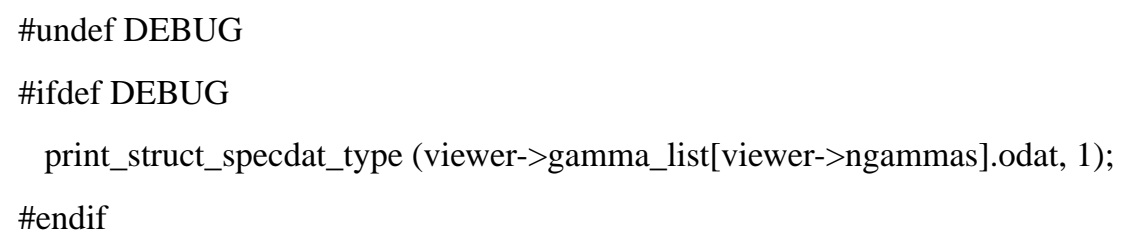


/

$* * * * * * * * * * * * * * * * * * * * * * * * * * * * * * * * * * * * * * * * * * * * * * * * * * * * * * * * * * * * * * * * * * * * * * *$ $* * * * * * *$

* write_cdf_waveform() writes wave data to an ascii CDF file.

* A CDF header is followed by bin edges \& flux pairs.

* 15 jun 1993 clh create from write_waveform

* 24 aug 1993 clh change CDF output file data format; add FILETYPENO \& TIMECAL

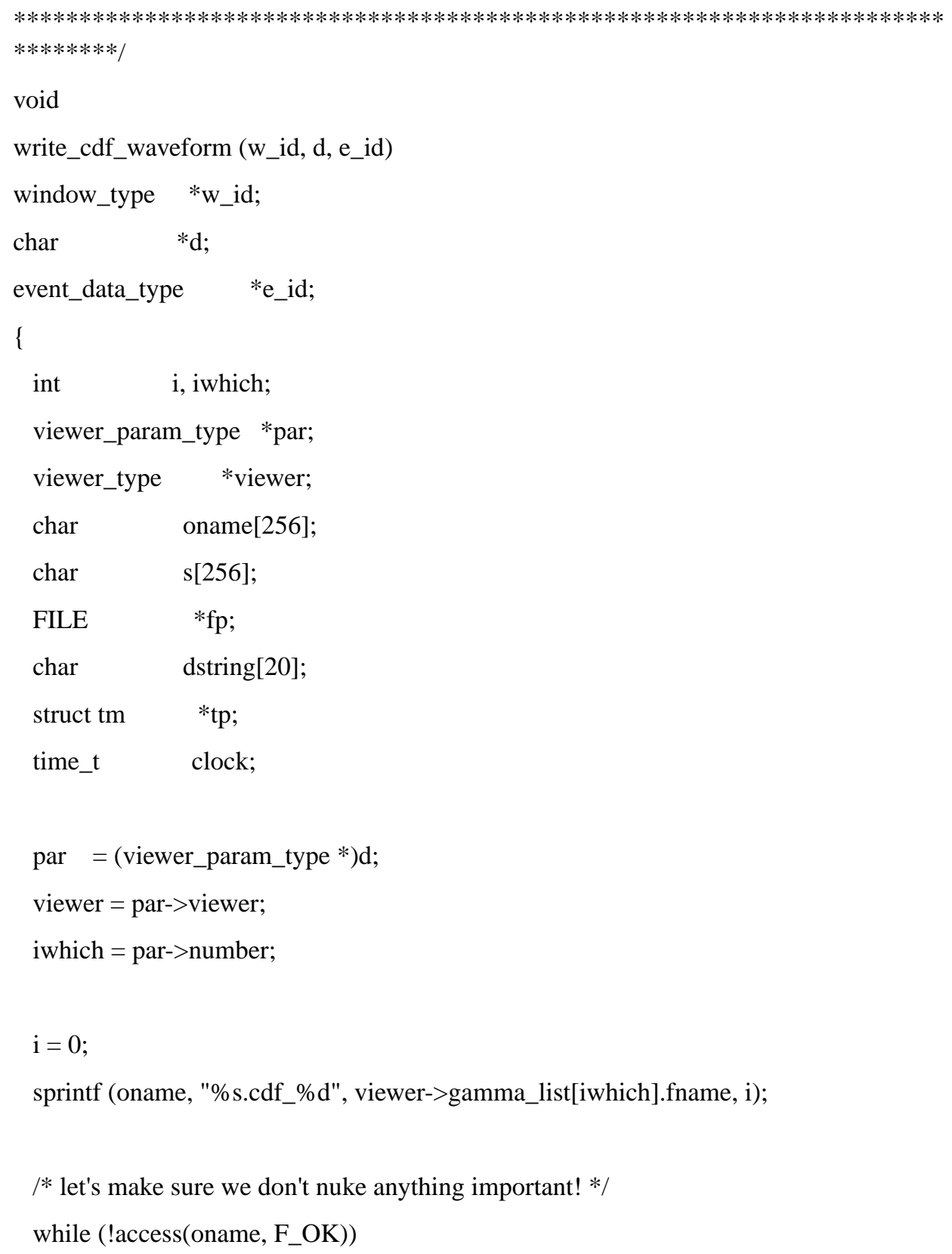




\section{Appendix A: An Introduction to the Common Data Format (CDF)}

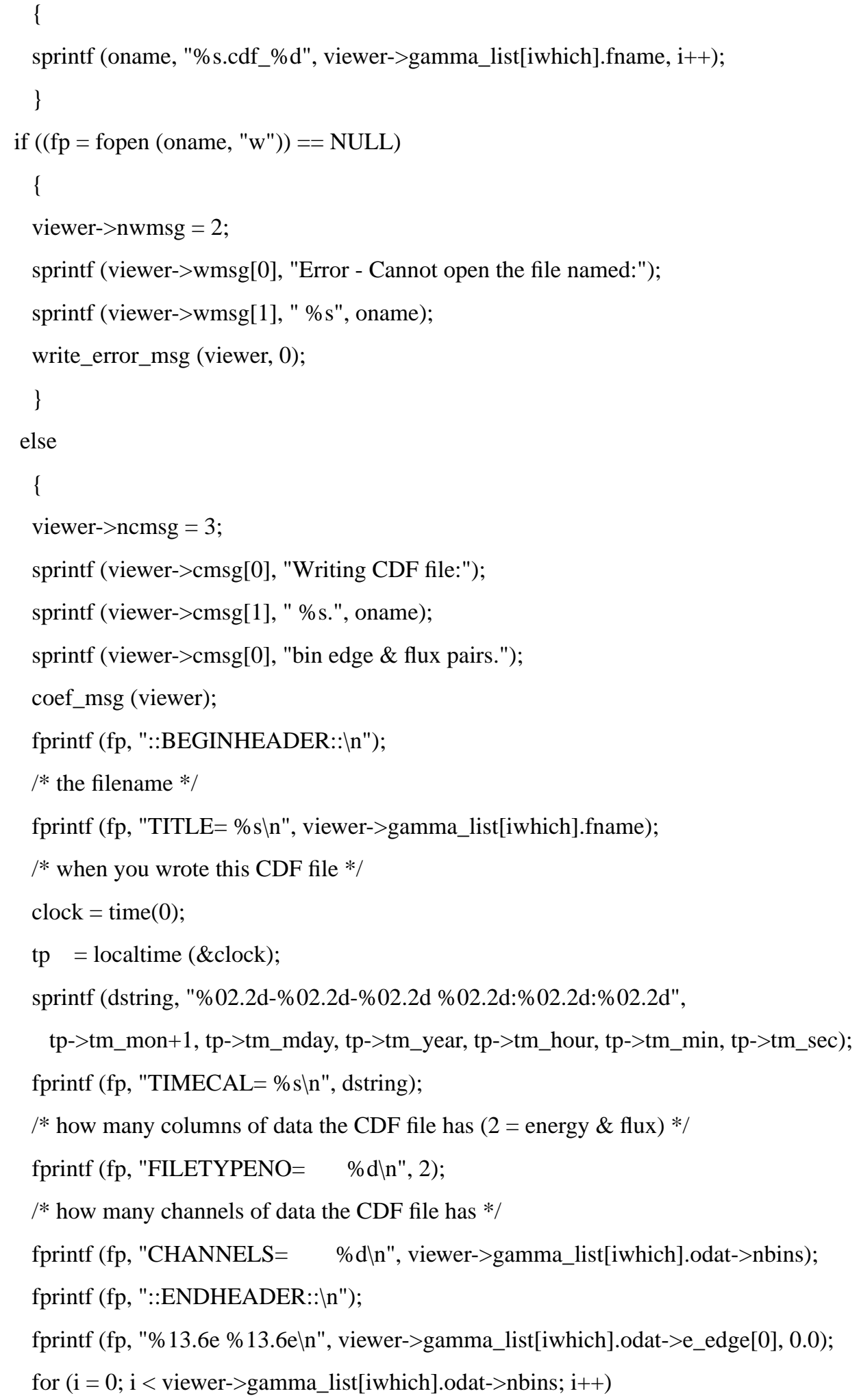




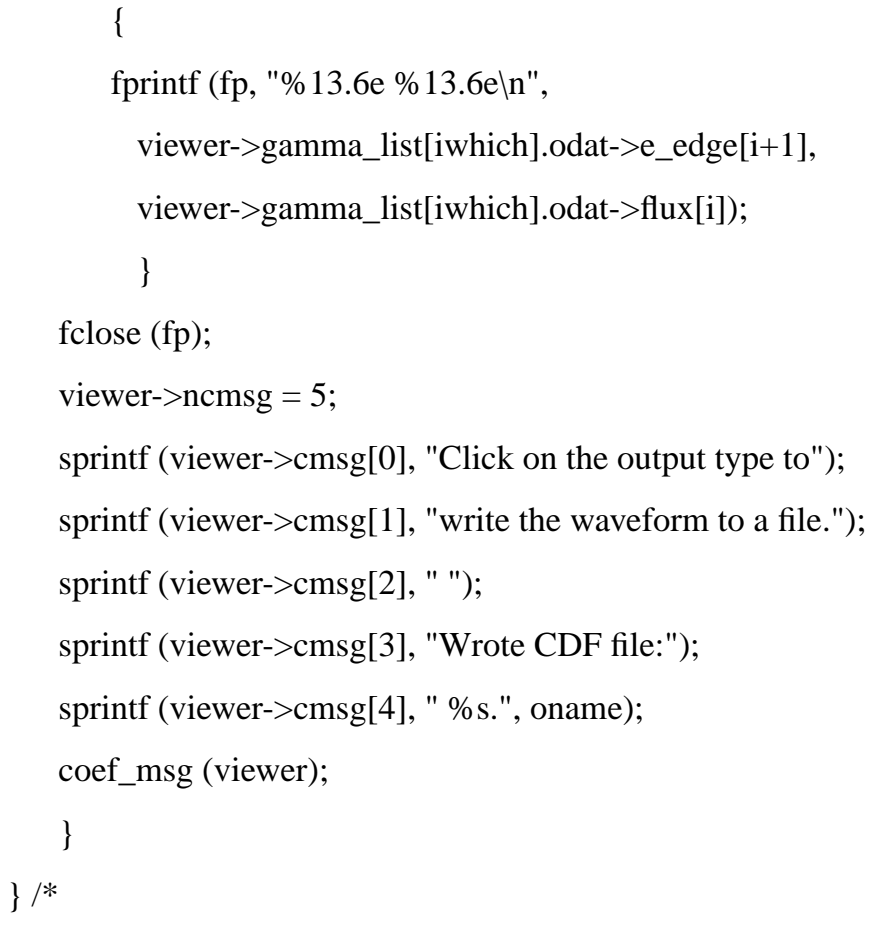




\section{Appendix B: About HDF and supported platforms}

In this appendix are a few web pages downloaded from the National Center for Supercomputing Applications (NCSA) web site, http://hdf.ncsa.uiuc.edu/, that define the Hierarchical Data Format (HDF) and list the platforms (with compiler information) on which the DCSA HDF group tested HDF and for which pre-compiled binaries are provided on the NCSA ftp server. 